\title{
CIRSE Guidelines: Quality Improvement Guidelines for Endovascular Treatment of Traumatic Hemorrhage
}

\author{
Sam Chakraverty $\cdot$ Karen Flood $\cdot$ David Kessel • \\ Simon McPherson • Tony Nicholson • Charles E. Ray Jr. • \\ Iain Robertson • Otto M. van Delden
}

Received: 22 February 2011 / Accepted: 22 December 2011/Published online: 20 January 2012

(C) Springer Science+Business Media, LLC and the Cardiovascular and Interventional Radiological Society of Europe (CIRSE) 2012

\section{Introduction}

This quality improvement guideline outlines the place of interventional radiology (IR) in trauma management and indicates how imaging and IR can be used in the context of hemorrhage in the severely injured patient, and when IR is appropriate and when it is contraindicated. Vascular injury may also lead to occlusion, and this will be discussed where relevant. There is no intention for this document to be proscriptive; CIRSE and the writing committee recognize that successful centres may practice differently. The intention is to indicate how to develop the multidisciplinary linkages and infrastructure required for successful integration of imaging and IR in the trauma pathway. It is hoped that this will help prepare the way for greater consensus in the adoption of imaging and intervention in the management of the critically injured patient.

\section{S. Chakraverty}

Ninewells Hospital, Dundee, Scotland DD1 9SY, UK

K. Flood · D. Kessel ( $₫)$ - S. McPherson · T. Nicholson Leeds Teaching Hospitals, 1 Great George Street,

Leeds LSI 3EX, UK

e-mail: David.Kessel@leedsth.nhs.uk

C. E. Ray Jr.

University of Colorado Hospital, 12401 E 17th Ave,

Room 526, Mail Code L954, Aurora, CO 80045, USA

\section{Robertson}

Gartnaval General Hospital, 1053 Great Western Road, Glasgow, Scotland G12 0YN, UK

O. M. van Delden

Department of Radiology, Academic Medical Centre,

Meibergdreef 9, 1105, Amsterdam, Zuidoost, The Netherlands
The information presented here reflects available evidence and draws on pathways already in clinical use. It is intended for local consideration and adaptation according to current and future resources. Recommendations for practice and quality improvement are indicated throughout, and a checklist for safe use of intervention in the management of traumatic hemorrhage is included. Wherever possible, levels of evidence and grades of recommendation are based on those proposed by Oxford Centre for Evidence-based Medicine.

A fuller version of this document is available at the CIRSE Web site.

\section{Basic Trauma Management}

\section{Resuscitation}

Resuscitation begins at the scene of the accident and continues throughout the process of transfer, admission to hospital, triage, further investigation, and treatment. The immediate aim of trauma care is to ensure adequate oxygenation by treating airway and ventilation problems and supporting circulation. This initial phase of resuscitation reduces early death and helps to prevent long-term problems and late mortality due to multiorgan failure and sepsis.

Hemorrhage is the major preventable cause of early trauma death; hypotension also results in organ hypoperfusion, which increases late death and long-term disability. Hemorrhage may be self-limiting, may be stopped by external pressure, or may require IR or surgery to control. The total time from patient injury to stopping hemorrhage critically affects outcome.

Imaging should be performed before radiologic or operative intervention in patients who respond at least 
partially to resuscitation. Patients with ongoing hemodynamic instability that does not respond at least partially to adequate resuscitation may require immediate intervention. This must be the trauma team leader's decision. Other lifesaving imaging and interventions should not be delayed until (or if) the patient becomes hemodynamically stable.

Recommendation 1 Approximately 50\% of trauma occurs outside of working hours; therefore, the emergency department senior team leader and other services, including computed tomographic (CT) imaging and IR, should be available $24 \mathrm{~h}$ a day. Patients should only be taken to appropriately staffed and resourced facilities.

\section{Transfers}

There are typically several stages of early care for the severely injured patient, which will involve several teams and transfers to more than one location. For this to be safe, the anesthetic and emergency medical services need to be as familiar with the CT and angiography suites as they are with the emergency operating theatre. This may require rehearsal of transfers from the emergency department to and from the $\mathrm{CT}$ and angiography suites and the operating theatre. $\mathrm{CT}$ and angiography must be adequately equipped to allow resuscitation and other management to continue during imaging and radiologic/surgical intervention. This should produce a pathway where consultant-level opinion is provided and the patient quickly transferred to and from CT with the aim of minimizing time to achieve hemostasis.

Recommendation 2 Each centre must devise, rehearse, and regularly review arrangements for effective resuscitation by appropriately skilled and equipped teams during transfers within the hospital and during the investigation and treatment of these patients.

\section{Subsequent Care}

The final phase of trauma care is provided by specialist teams that focus on damage repair and prevention of longterm disability. It is important to recognize that IR may also contribute to damage repair, particularly in terms of revascularisation of ischemic injury. IR also plays a role in management of ureteric and biliary injury and drainage of collections. These are not the prime focus of immediate trauma care and will not be discussed further.

\section{Imaging}

Multidetector computed tomography (MDCT) has revolutionized the diagnosis and treatment of trauma patients, identifying the vast majority of injuries in a single and rapid study. Multiplanar reformatted images can guide the appropriate use of IR or surgery in trauma by identifying sites and causes of recent or active bleeding. Appropriate scrutiny of CT images will often reveal structural abnormalities, such as transection, dissection, false aneurysm, and arterial cutoff, all of which relate to significant vascular injury.

Level 3 evidence suggests that early whole body CT improves outcome, and that MDCT can shorten the criterion time and can indicate whether hemostatic control is best achieved by nonoperative management (NOM), IR, or damage control surgery (DCS). Resuscitation can and should continue in modern CT environments. The ongoing REACT II trial will, in the future, provide level 1 evidence on the effect of a CT-first protocol.

If the CT scanner is not in the emergency department, then assessment should define what is possible within existing architectural, staffing, and transport limitations. In every case, the optimal service should be achieved by using existing resources.

Focused abdominal sonography in trauma (FAST) and plain films (chest, cervical spine, pelvis) do not add anything to the information provided by a CT scan. In unstable patients in civilian settings, FAST is a poor discriminator for those patients requiring laparotomy, with negative predictive values of only $50-63 \%$.

Recommendation 3 Whole body MDCT (head to midthighs/knees) should be the default first-line imaging in severely injured patients who respond at least partially to resuscitation. MDCT should be available within $30 \mathrm{~min}$ of requests and should be performed before angiography or surgery. Other investigations should not delay CT. In very unstable patients, CT should follow immediate surgery or balloon occlusion to control bleeding. (Recommendation B, level 2 evidence.)

\section{Therapeutic Options}

The total time from patient injury to stopping hemorrhage is crucial in affecting outcome. Controlling bleeding will only be achieved if coagulopathy is minimized by appropriate blood product support and drug therapy. NOM is preferred if possible, but many cases require DCS, IR techniques, or a combination of the two. IR and DCS are complementary techniques.

Recommendation 4 IR and DCS should be recognized as complementary techniques. IR should be the minimally invasive component of a complete damage control management armamentarium. 


\section{Evidence for DCS and IR in Trauma}

Few aspects of immediate trauma care are founded on the basis of high-quality prospective research. IR is no exception. Because of the heterogeneity of the presenting population, it is difficult to envisage level 1 evidence being achieved. Most evidence relating to DCS and IR is based on retrospective cohort studies (level 3 evidence), which have inherent limitations. With this caveat, we note that there are no major contradictory findings to the use of IR to arrest hemorrhage in major trauma.

Despite the attraction of a minimally invasive approach, IR is not always the best (or most rapid) treatment. Choice of therapy should be determined by senior clinicians on the bases of the constellation of injuries present in each patient and the local expertise available.

Decisions on embolization for traumatic hemorrhage are typically made by both the trauma team leader/surgeon and the interventional radiologist, and should be made quickly on an individual-patient basis according to locally agreed algorithms. Establishing routes of communication between the services is paramount. The final decision needs to reflect the resources available at any given institution.

Whichever investigation or therapy is chosen, clear protocols must detail who is responsible for patient care throughout. An example is shown in Appendix 1.

Recommendation 5 Each unit must develop and agree on interdepartmental protocols delineating responsibility for the management of every stage of the patient journey from arrival to intervention.

Centres providing care for major trauma should aim to offer the full spectrum of NOM, DCS, and IR according to the clinical need of each patient. Not every hospital will be able to provide comprehensive care for trauma. It is essential that the emergency services know which services are appropriately equipped. In general, severely injured patients should be taken directly to these sites even if this involves a longer transfer. Secondary transfer should be avoided.

Recommendation 6 Because IR can increase the proportion of patients treated by NOM, these techniques should be promoted, provided suitable IR skills exist to perform embolization, stent grafting, or balloon occlusion to quickly control bleeding. (Recommendation C, level 3 evidence. This is equivalent to the evidence for surgical management; see Appendix 2.)

\section{Options for IR Involvement}

IR should be considered in terms of the techniques available and how they may be used in a variety of clinical scenarios. In the context of trauma, vascular interventional radiologists may need to stop hemorrhage and/or restore blood flow.

\section{Controlling Bleeding}

Percutaneous transcatheter embolization is the most frequent treatment. Permanent (coils, plugs, polyvinyl acetate, glue) or temporary (Gelfoam/Spongostan, thrombin) materials are appropriate in different situations. Covered stent grafts can also be used in major vessel injury and suitably sized vessels where preservation of flow is desirable. Appropriate equipment should be readily available and stocked in sufficient quantity in the angiography department.

In some circumstances, the only IR contribution is balloon occlusion of thoracic or infrarenal aorta. Clinicians should be aware of techniques for insertion of these and note that on occasion this may be necessary without fluoroscopic guidance.

All institutions must work within the limits of their setting. An example of an algorithm that may be used in the treatment of traumatic pelvic hemorrhage is presented in Appendix 3. Such algorithms or key clinical pathways are usually beneficial as a guideline for treatment methods that require more than one clinical service.

Suggestions for the choice of NOM, IR, and DCS are provided in Appendix 2. The patient's clinical condition will also affect decision making. In every case, close clinical review and discussion before, during, and after IR procedures is essential because situations can evolve rapidly.

\section{Managing Ischemia}

Significant arterial injuries can cause distal ischemia as a result of spasm, dissection, thrombotic occlusion, and even complete arterial transection. In most cases, restoration of flow is achieved by stenting or stent grafting. Where there is thrombus, consideration needs to be given to mechanical thrombectomy techniques and thrombus aspiration. Bleeding almost invariably takes precedence over ischemia.

Details of technique and the choice of agent are beyond our scope here.

\section{Aorta and Peripheral Arteries}

\section{Thoracic Aorta}

Injuries to the thoracic aorta set the standard for IR treatment in trauma. 


\section{Techniques}

Thoracic endovascular aneurysm repair (EVAR) is the technique used to treat injuries to the thoracic aorta.

\section{Indications for IR}

One indication for IR includes injury to the thoracic aorta distal to the left subclavian artery. Aortic injury is usually asymptomatic, in which case treatment is to prevent rupture. Active bleeding is rare, as is distal ischemia due to pseudocoarctation or dissection. Some small partial-thickness tears can be observed or treated electively.

\section{Exclusions}

Injuries to the ascending aorta are managed by cardiothoracic surgery. Injuries to the arch adjacent to or involving the great vessels will usually require either surgery or sometimes a joint approach with bypass surgery to ensure cerebral circulation.

\section{Outcomes}

Endovascular repair is associated with improved survival compared to open repair of traumatic aortic injury (mortality 7.6 vs. $15.2 \% P=0.008$ ). Survival after endovascular repair depends on the associated injuries. There is a significant reduction in all major treatment complications, including paraplegia and stroke.

Recommendation 7 Stent grafting should be used in the management of most patients with thoracic aortic injury distal to the left subclavian artery. (Recommendation B, level 2a.)

\section{Abdominal aorta}

\section{Techniques}

Balloon occlusion to control infra-aortic hemorrhage and stent grafting are used to treat injuries to abdominal aorta.

\section{Indications}

Localized aortic injury and distal hemorrhage are indications for treatment.

\section{Exclusions}

Stent grafting should not be performed if it would lead to the occlusion of vital branch vessels to the kidneys and gastrointestinal tract.

\section{Outcomes}

Aortic balloon occlusion is appropriate if it can be achieved more quickly than surgical control. A few localized injuries can be treated with stent grafts. There is insufficient evidence to make formal recommendations for either surgery or IR.

Recommendation 8 Management of abdominal aorta should be decided on a case-by-case basis according to local expertise. (Recommendation C, level 4.)

Peripheral and Visceral Arteries

\section{Techniques}

Treatment for peripheral and visceral arteries includes the following: aortic or proximal balloon occlusion in sites inaccessible to tourniquet to control bleeding; stent graft for transection, false aneurysm, and arteriovenous fistula; stent or stent graft for arterial occlusion; embolization for focal bleeding if the vessel can safely be sacrificed (e.g., profunda femoris artery, most branch arteries).

\section{Indications}

Indications for treatment include blunt and penetrating peripheral arterial injury with contrast extravasation, false aneurysm, arteriovenous fistula, significant tears in the lining, and significant distal ischemia.

\section{Exclusions}

Revascularization should not be performed when there is nonviable tissue.

\section{Outcomes}

There is insufficient evidence to make formal recommendations for either surgery or IR. Stent grafting and embolization may be definitive treatments and may spare the patient further operative intervention.

Recommendation 9 Treatment of injuries to peripheral and visceral arteries should be decided on a case-by-case basis according to local expertise. (Recommendation C, level 4.)

\section{Solid Abdominal Organs}

Spleen

\section{Indications}

Indications for treatment include splenic injury with active arterial bleeding or false aneurysm, and splenic artery injury. 


\section{Techniques}

Techniques to treat injuries to the spleen include stent graft for injury to main splenic artery; proximal embolization for diffuse splenic injury; and distal selective embolization for focal bleeding or false aneurysm.

\section{Outcomes}

There is no difference in mortality (6-11\%) between IR and splenectomy in patients with traumatic injury to the spleen.

\section{Splenic Salvage}

Splenectomy is associated with an increased risk of developing overwhelming sepsis. IR preserves splenic function and reduces the rate of splenectomy. The benefits of embolization are much higher in severe (grade IV) splenic injuries, where splenic salvage is seen in $84-94 \%$ of patients treated with embolization, compared to $4 \%$ if assigned to NOM.

\section{Complications}

Complications of embolization occur in up to $15 \%$ of patients and include recurrent hemorrhage and abscess formation. Infection in the first 30 days after splenectomy is reported in up to $45 \%$ of patients.

Recommendation 10 Proximal or selective embolization of the spleen can preserve immune competence and should be offered for active arterial bleeding and false aneurysm. (Recommendation C, level 3.)

\section{Liver}

\section{Indications}

Intervention or surgery is indicated for focal active arterial bleeding (without portal venous injury), ongoing arterial hemorrhage, or false aneurysm after damage-control surgery.

\section{Exclusions}

Hemodynamically stable patients without active bleeding or false aneurysm do not require intervention. Even high-grade liver injuries can be managed by NOM. Embolization in the presence of portal vein occlusion will lead to infarction.

\section{Techniques}

Selective embolization is used to treat focal bleeding or false aneurysm; proximal embolization is used to treat diffuse arterial injury.

\section{Outcomes}

Arterial hemorrhage from the liver is difficult to treat with surgery; packing is usually the only surgical option. Partial hepatectomy is associated with a very high mortality.

\section{Survival}

IR can reduce the rates of laparotomy, but there is no evidence of a difference in mortality between IR and DCS if used for the above indications.

\section{Complications}

High-grade liver injuries have significant complication rates that can theoretically be exacerbated by surgical or radiologic treatment. The number of complications such as infected hematoma or biloma are similar in patients with comparable injury grades who do not undergo embolization.

Recommendation 11 Proximal or selective embolization should be offered for active arterial bleeding and false aneurysm as an alternative to surgery or in cases where surgery has failed. (Recommendation C, level 4.)

\section{Kidney}

\section{Techniques}

Proximal embolization is used to treat diffuse injury, and selective embolization is used for focal bleeding points or false aneurysms.

\section{Indications}

Treatment is indicated in cases of active arterial bleeding, arteriovenous fistula, and false aneurysm or hematuria.

\section{Survival}

In patients selected for this procedure, survival is good. IR can preserve renal function, although this is not the primary aim of treatment. Hemostasis takes precedence in all cases.

\section{Preservation of Kidney}

Delayed nephrectomy rates are $12 \%$. Posttreatment kidney function is not known.

Recommendation 12 Embolization should be used to manage renal hemorrhage, especially in cases of focal injury, where there is likely to be preservation of function. (Recommendation C, level 3.) 
Pelvis

\section{Technique}

Treatment includes selective embolization and Gelfoam with or without embolization for multiple sites of arterial injury. Proximal embolization and internal iliac arterial ligation alone are less effective.

\section{Indications}

Patients who demonstrate arterial bleeding on CT or who are hemodynamically unstable despite pelvic wrap are candidates for treatment. Approximately 1-2\% of patients with pelvic fracture are hemodynamically unstable, and only one in 5 of these will be due to arterial bleeding. When emergency pelvic packing is used to treat arterial hemorrhage, subsequent IR may also be necessary. External fixation is only effective at controlling bleeding from bony and venous injury; if used in arterial bleeding, it compromises the performance of embolization.

\section{Outcomes}

Embolization is highly effective at stopping arterial bleeding in the context of pelvic injury.

\section{Complications}

For both surgical and IR treatments, complications are difficult to discriminate from those caused by the injury itself. Gluteal necrosis has been described after pelvic embolization. Sexual dysfunction frequently occurs after pelvic trauma, although its incidence is not obviously increased by embolization.

Recommendation 13 Embolization should be used to manage arterial bleeding resulting from pelvic trauma and should precede treatment other than pelvic binder. (Recommendation $\mathrm{C}$, level 3.)

\section{Developing an IR Trauma Service}

\section{Regional Environment}

Improvements in clinical outcomes require the development of robust trauma networks that optimize access across the region. A trauma network comprises all stakeholders involved in the care of the severely injured patient, starting with the ambulance services and continuing to acute clinical care and rehabilitation centres.
Levels of Service

Some hospitals within a region will be designated major trauma centres, capable of dealing with the most severely injured patients. This designation should be based on the range of services provided and a minimum number of patients. Outcomes relate to quality of the facilities, the skill of staff members, and staff seniority. The level of services and IR support in adjacent centres need to be agreed and defined to ensure prompt appropriate transfer and treatment. The major trauma centre should provide leadership and act as an enabler to allow adjacent centres to develop and deliver appropriate levels of service.

Major trauma centres should treat the most severely injured patients. They must provide multiple clinical, diagnostic, surgical, and anesthetic services, including access to cardiothoracic and neurosurgical units $24 \mathrm{~h}$ a day. The development of rapid patient pathways is vital to the coordination of acute clinical care across specialties, and imaging departments must be part of this.

Whenever new facilities are being developed, imaging and angiography suite room sizes, anaesthetic facilities, and sterility standards appropriate for use in trauma patients must be considered.

As trauma networks develop, patients will be triaged to appropriate centres, which may result in patients bypassing smaller units. When direct transportation to the major centre is not possible, smaller units will need to stabilize patients with major trauma, and they need to have a plan in place to deliver these services. Smaller hospital imaging departments may therefore have to consider the provision of CT and, less commonly, IR support for this patient group as part of the trauma network.

Recommendation 14 Services should assess themselves according to the requirements for IR as set out in Appendix 4. Those involved in managing major trauma must be able to fulfil all the listed criteria.

Local Imaging Requirements

Rapid access to CT after initial resuscitation is essential. Local departmental geography will vary; however, CT should ideally be located immediately adjacent to or within the emergency department.

Interventional Radiology

\section{Angiography Suites}

Rapid access to angiographic intervention is essential. Not every angiography suite is suitable to handle the difficulties that may arise from performing trauma interventions. Ideally, angiography suites should be collocated within an 
acute-care theatre complex that will provide surgical and anaesthetic support to acutely ill patients. Angiographic equipment located in a theatre-grade environment can support both open surgery and endovascular IR procedures.

\section{Angiographic Hardware}

Angiography suites must have high-end imaging equipment and need to be large enough to handle the numerous individuals who accompany very unstable trauma patients. Modern fixed $\mathrm{C}$-arm angiographic equipment offers the best imaging to support IR and deliver rapid arrest of hemorrhage, and it is essential when IR is to be involved in trauma.

Portable C-arm equipment should only be used in the context of immediate stabilization by occlusion balloon inflation. Portable units do not offer the same imaging quality as fixed units, and there is evidence of patient harm occurring with the use of such units, principally as a result of poor image quality. In addition, portable units can only operate for a short time before overheating.

\section{Protocols}

Predetermined protocols within the department for transfer, imaging, and intervention are essential to ensure a consistent and auditable quality of care.

Local services should take particular care to develop transfer protocols for both internal and external anaesthetic-supported transfer. A frequent source of delay in many centres is the internal transfer of metastable patients for CT imaging or embolization. Agreed pathways and improvements to local environments should be prioritized to minimize delay while maintaining patient safety.

\section{Workforce}

Trauma frequently occurs outside normal working hours, and the best clinical outcomes are achieved by rapid access to a consultant- or attending physician-led and -delivered service. In some centres, this will pose a particular challenge for imaging and intervention because the required staffs are not resident on call.

The development of early warning systems for on-call IR teams is strongly advised. The priority must always be to develop systems that reduce the key clinical criterion of total time to arrest hemorrhage.

\section{Consumable Equipment}

The priority in most IR cases is to arrest hemorrhage quickly. The use of embolization packs or bags that contain a selection of occlusion balloons, catheters, embolic materials, and stent grafts may reduce the time to treatment. These packs are particularly recommended when procedures are being undertaken outside the routine angiographic environment.

\section{Audit}

Multidisciplinary team audit including all involved specialties is essential to improve and maintain high-quality clinical services. Radiologists should ensure that they participate in ongoing audits of trauma services and contribute to local and national audit mechanisms.

Recommendation 15 All IR trauma services must continue to ensure that they meet the specifications for an effective and safe IR service (Appendix 4). They must audit their results against nationally prescribed standards.

Conflict of interest The authors declare that they have no conflict of interest.

\section{Appendix 1}

Responsibility for the hemodynamically compromised patient after blunt truncal trauma. 


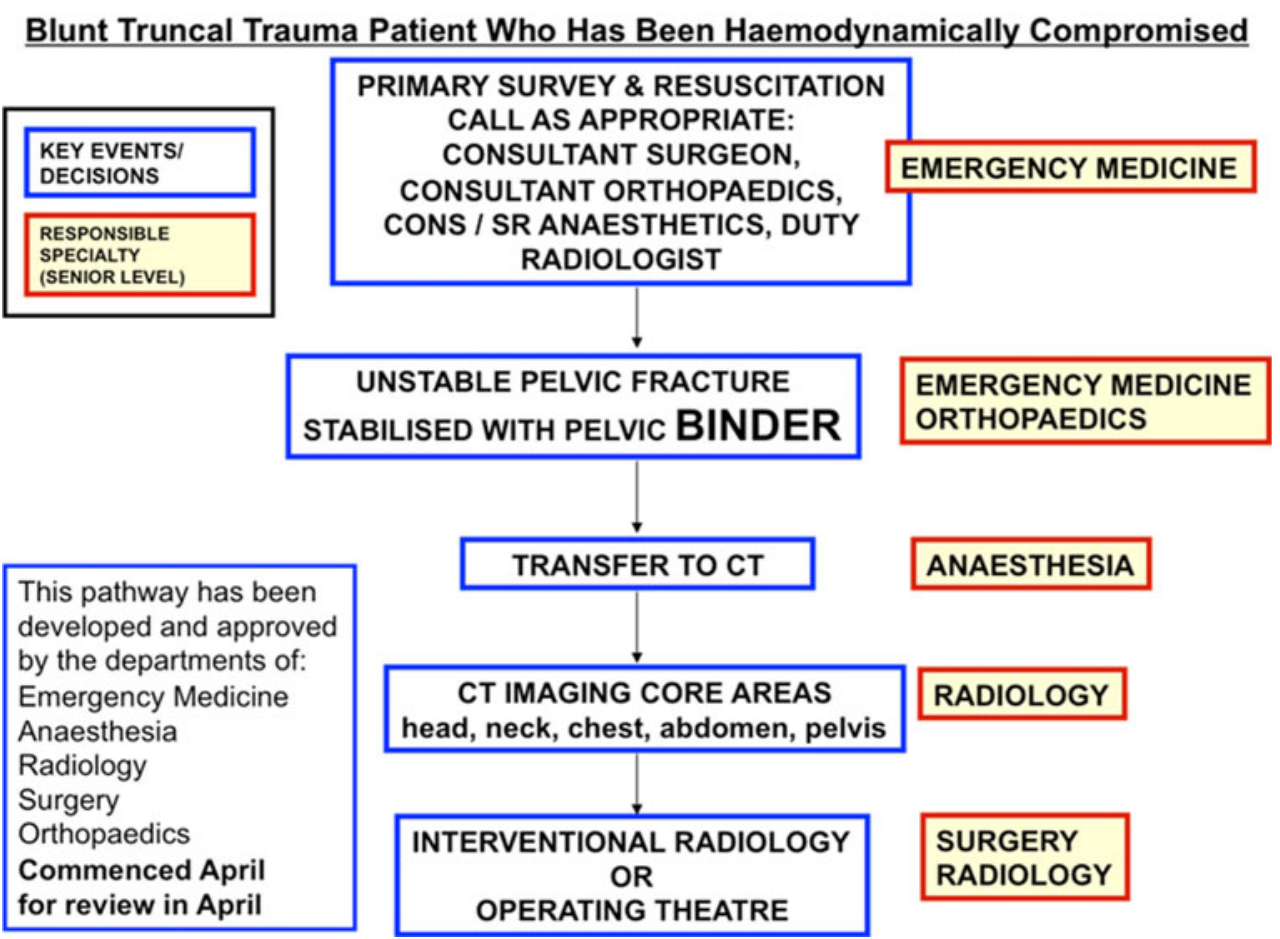

\section{Appendix 2}

Outline indicating how decision making might be influenced by nature of injuries

\begin{tabular}{|c|c|c|c|}
\hline Site & Nonoperative management & Interventional radiology & Damage control surgery \\
\hline Thoracic aorta & $\begin{array}{l}\text { No role except in small } \\
\text { partial thickness } \\
\text { tears }(4, \mathrm{C})\end{array}$ & Stent graft for suitable lesions $(2, \mathrm{~B})$ & $\begin{array}{l}\text { Ascending aortic injury or arch injury } \\
\text { involving great vessels }(4, \mathrm{C})\end{array}$ \\
\hline Abdominal aorta & No role & $\begin{array}{l}\text { Occlusion balloon, stent graft for } \\
\text { suitable lesions }(4, C)\end{array}$ & $\begin{array}{l}\text { Injury requiring visceral revascularization } \\
\text { or untreatable by endovascular therapy } \\
(4, C)\end{array}$ \\
\hline $\begin{array}{l}\text { Peripheral or } \\
\text { branch artery }\end{array}$ & No role & $\begin{array}{l}\text { Occlusion balloon, stent, stent graft } \\
\text { or embolization }(4, C)\end{array}$ & $\begin{array}{l}\text { Any lesion that cannot rapidly be } \\
\text { controlled or that will require other } \\
\text { revascularization }(4, C)\end{array}$ \\
\hline Kidney & $\begin{array}{l}\text { Subcapsular or } \\
\text { retroperitoneal hematoma } \\
\text { without active arterial } \\
\text { bleeding }(3, \mathrm{C})\end{array}$ & $\begin{array}{l}\text { Active arterial bleeding, embolization } \\
\text { or stent graft }(4, \mathrm{C}) \text {; arterial } \\
\text { occlusion }<6 \mathrm{~h}, \text { stent/stent graft }\end{array}$ & $\begin{array}{l}\text { Renal injury in association with multiple } \\
\text { other bleeding sites, or other injuries } \\
\text { requiring urgent surgical repair }\end{array}$ \\
\hline Spleen & $\begin{array}{l}\text { Lacerations, hematoma } \\
\text { without active bleeding, } \\
\text { evidence of false } \\
\text { aneurysm }(3, \mathrm{C})\end{array}$ & $\begin{array}{l}\text { Active arterial bleeding or false } \\
\text { aneurysm; focal embolization } \\
\text { for focal lesion; proximal } \\
\text { embolization for diffuse injury } \\
(3, \mathrm{C})\end{array}$ & $\begin{array}{l}\text { Packing or splenectomy for active } \\
\text { bleeding in association with multiple } \\
\text { other bleeding sites }\end{array}$ \\
\hline Liver & $\begin{array}{l}\text { Subcapsular or } \\
\text { intraperitoneal hematoma, } \\
\text { lacerations without active } \\
\text { arterial bleeding }(3, \mathrm{C})\end{array}$ & $\begin{array}{l}\text { Active focal arterial bleeding; focal } \\
\text { embolization if possible; } \\
\text { nonselective embolization if } \\
\text { multiple bleeding sites as long as } \\
\text { portal vein patent }(3, \mathrm{C})\end{array}$ & $\begin{array}{l}\text { Packing if emergency laparotomy is } \\
\text { needed, with subsequent repeat CT and } \\
\text { embolization if required }\end{array}$ \\
\hline Pelvis & $\begin{array}{l}\text { Minor injury with no active } \\
\text { bleeding }\end{array}$ & $\begin{array}{l}\text { Focal embolization for arterial injury } \\
\text { (bleeding, false aneurysm or cutoff) } \\
(3, \mathrm{C})\end{array}$ & $\begin{array}{l}\text { External compression and subsequent } \\
\text { fixation if bleeding from veins or bones }\end{array}$ \\
\hline
\end{tabular}


Appendix 2 continued

\begin{tabular}{llll}
\hline Site & Nonoperative management & Interventional radiology & Damage control surgery \\
\hline Intestine & Focal contusion with no & Focal bleeding with no evidence of & Ischemia or perforation requiring \\
& evidence of ischemia, & ischemia or perforation, or to & laparotomy with or without bowel \\
& perforation, or & stabilize the patient, allowing & interval laparotomy pending \\
hemorrhage (3, C) & treatment of other injuries (4, D) &
\end{tabular}

The patient's clinical condition will also affect decision making. Level of evidence: 1, 2, 3, 4, 5; grade of recommendation: A, B, C, D

\section{Appendix 3}

Algorithm for treatment of traumatic pelvic hemorrhage.

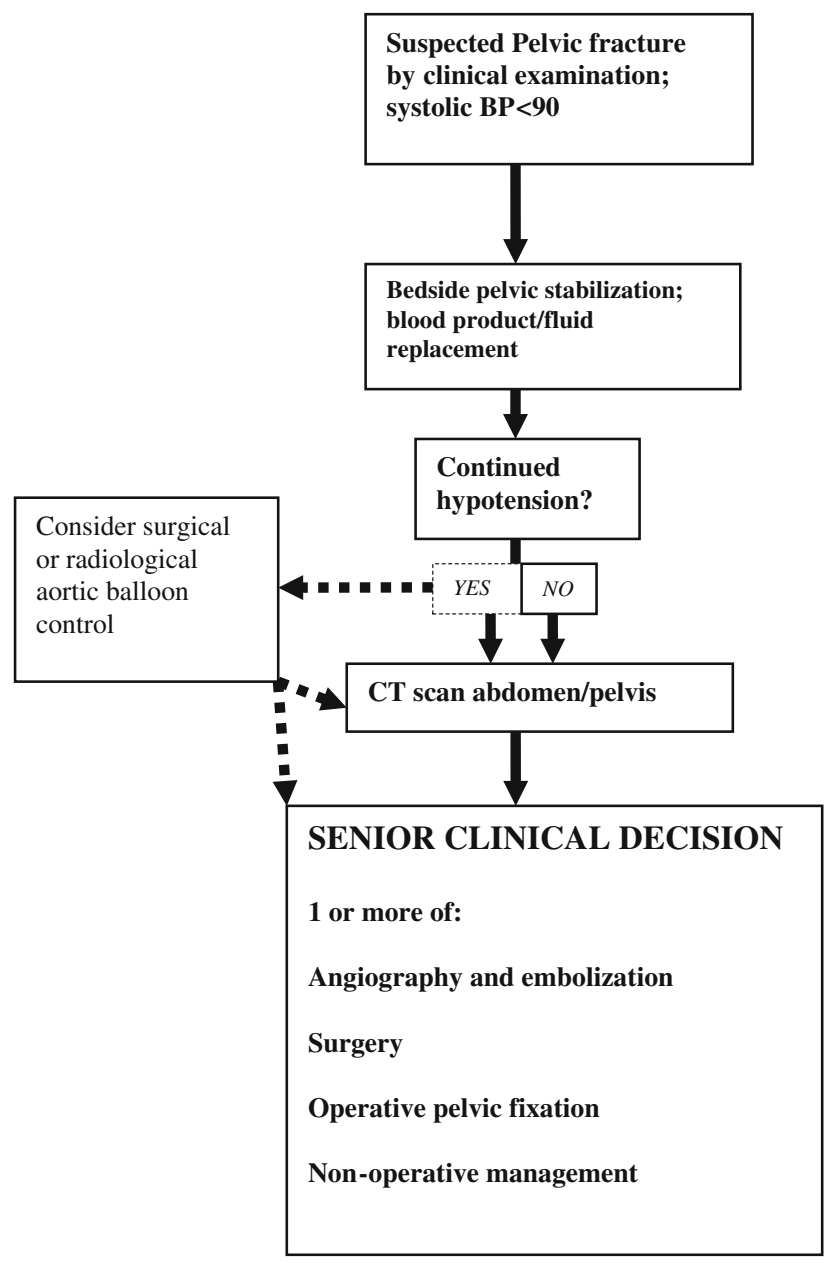

\section{Appendix 4}

Checklist for safe use of intervention in the management of traumatic hemorrhage

Assessing imaging equipment, consumables, and staffing $\quad \mathrm{Y} / \mathrm{N}$

Imaging $^{\mathrm{a}}$

- Multislice CT scanner staffed and available $24 \mathrm{~h}$ per day. CT imaging should be available within $30 \mathrm{~min}$ of a trauma call

- Modern angiography equipment with at a minimum a fixed $\mathrm{C}$-arm and digital subtraction

- Contingency plans exist to cover routine service and breakdown

- CT and angiography unit housed within the trauma suite (ideal)

Consumables $^{\mathrm{b}}$

The following must be available at all times:

- Full range of angiographic sheaths, guide catheters, catheters and guide wires including microcatheters and coil pushers

- Full range of embolic agents: coils (including microcoils), vascular plugs, Gelfoam (occasionally glue or polyvinyl acetate)

- Occlusion balloons of various sizes to allow aortic and iliac occlusion

- Stent grafts of various sizes to allow treatment of thoracic aortic injury and peripheral and visceral vascular injury

- A trauma box should be maintained with all necessary kit readily available (ideal)

Staffing $^{\mathrm{c}}$

The minimum requirement is for the following staff to be available at all times:

- CT radiographer

- Interventional radiologist capable of embolization and stent grafting

- Angiography radiographer 
Appendix 4 continued

Assessing imaging equipment, consumables, and staffing $\mathrm{Y} / \mathrm{N}$

- Angiography nurse

Portering

- The angiography team should be mobilized as soon as there is a major trauma call to allow embolization to start within minutes of diagnosis being established (ideal)

Protocols

- Locally agreed protocols and management pathways should exist for the investigation and treatment of hemorrhage in the severely injured patient. There must be clear delineation of clinical and decision making responsibility for each stage

- Transfer and resuscitation of patients to and from the imaging department and angiographic suite

- Transfusion, replacement of blood products and correction of coagulopathy

Clinical scenarios

Locally agreed strategy for the use of intervention in the management of hemorrhage (or prevention of bleeding) in the following clinical scenarios must be clear:

- Pelvic fracture

- Solid organ injury

- Blunt aortic injury

${ }^{a}$ Equipped with piped gases and anaesthetic equipment as locally specified

b An individual or individuals must be responsible for stock management stock levels must be adequate (there needs to be some redundancy), checked regularly, and items replaced when levels are low or out of date

c The on-call rota must be formal, robust, sustainable and sufficiently attractive to allow staff recruitment and retention

If the answer to any of the above questions is no, then the service is not equipped to manage major trauma. This should be detailed in the hospital risk management strategy, and arrangements to provide suitable alternatives should be made

\section{Appendix 5: Recommended Reading}

\section{Overviews}

National Confidential Enquiry into Patient Outcome and Death (2007) Trauma: who cares? http://www.ncepod.org. uk/2007t.htm

NHS Clinical Advisory Group (2010) Regional networks for major trauma. http://www.dh.gov.uk/en/Publications andstatistics/Lettersandcirculars/Dearcolleagueletters/DH_ 120048

\section{Resuscitation}

Jansen JO, Thomas R, Loudon MA, Brooks A (2009) Damage control resuscitation for patients with major trauma. BMJ 338:b1778
Focused Abdominal Ultrasound in Trauma

Friese RS, Malekzadeh S, Shafi S et al (2007) Abdominal ultrasound is an unreliable modality for the detection of hemoperitoneum in patients with pelvic fracture. J Trauma 63:97-102

Körner M, Krötz MM, Degenhart C et al (2008) Current role of emergency US in patients with major trauma. Radiographics 28:225-242

Lee BC, Ormsby EL, McGahan JP et al (2007) The utility of sonography for the triage of blunt abdominal trauma patients to exploratory laparotomy. AJR Am J Roentgenol 188:415-421

\section{CT in Trauma}

Fang JF, Wong YC, Lin BC et al (2006) Usefulness of multidetector computed tomography for the initial assessment of blunt abdominal trauma patients. World J Surg 30:176-182

Huber-Wagner S, Lefering R, Qvick LM et al; Working Group on Polytrauma of the German Trauma Society (2009) Effect of whole-body CT during trauma resuscitation on survival: a retrospective, multicentre study. Lancet 373(9673):1455-1461

\section{Interventional Radiology in Trauma}

Bühren V, Trentz O (1989) [Intraluminal balloon occlusion of the aorta in traumatic massive hemorrhage] [in German]. Unfallchirurg 92:309-313

Wallis A, Kelly MD, Jones L (2010) Angiography and embolisation for solid abdominal organ injury in adults-a current perspective. World J Emerg Surg 5:18

Zealley IA, Chakraverty S (2010) The role of interventional radiology in trauma. BMJ 340:c497

Thoracic Aortic Injury

Hong MS, Feezor RJ, Lee WA, Nelson PR (2011) The advent of thoracic endovascular aortic repair is associated with broadened treatment eligibility and decreased overall mortality in traumatic thoracic aortic injury. J Vasc Surg 53:36-42

Riesenman PJ, Farber MA, Rich PB et al (2007) Outcomes of surgical and endovascular treatment of acute traumatic thoracic aortic injury. J Vasc Surg 46:934-940

Steenburg SD, Ravenel JG, Ikonomidis JS et al (2008) Acute traumatic aortic injury: imaging evaluation and management. Radiology 248:748-762

Tang GL, Tehrani HY, Usman A et al (2008) Reduced mortality, paraplegia, and stroke with stent graft repair of 
blunt aortic transections: a modern meta-analysis. J Vasc Surg 47:671-675

Xenos ES, Abedi NN, Davenport DL et al (2008) Metaanalysis of endovascular vs open repair for traumatic descending thoracic aortic rupture. J Vasc Surg 48:13431351

Splenic Injury

Dent D, Alsabrook G, Erickson BA (2004) Blunt splenic injuries: high nonoperative management rate can be achieved with selective embolization. J Trauma 56:10631067

Duchesne JC, Simmons JD, Schmieg RE Jr et al (2008) Splenic angioembolization does not improve outcomes in treating blunt splenic injuries compared with splenectomy: a cohort analysis. J Trauma 65:1346-1351

Gaarder C, Dormagen JB, Eken T et al (2006) Nonoperative management of splenic injuries: improved results with angioembolisation. J Trauma 61:192-198

Kaseje N Agarwal S Burch M et al (2008) Short-term outcomes of splenectomy avoidance in trauma patients. Am J Surg 196:213-217

Rajani R, Claridge C, Yowler J et al (2006) Improved outcome of adult blunt splenic injury: a cohort analysis. Surgery 140:625-632

Shanmuganathan K, Mirvis SE, Boyd-Kranis R (2000) Nonsurgical management of blunt splenic injury: use of CT criteria to select patients for splenic arteriography and potential endovascular therapy. Radiology 217:75-82

Wiseman J, Brown CV, Weng J et al (2006) Splenectomy for trauma increases the rate of postoperative infection. Am Surg 72:947-950

Hepatic Injury

Dabbs D, Stein DM, Scalea TM (2009) Major hepatic necrosis: a common complication after angioembolization for treatment of high grade liver injuries. J Trauma 66:621-629

Gaarder C, Naess PA, Eken P et al (2007) Liver injuries-improved results with a formal protocol including angiography. Injury 38:1075-1083
Misselbeck TS, Teicher EJ, Cipolle MD et al (2009) Hepatic angioembolization in trauma patients: indications and complications. J Trauma 67:769-773

Monnin V Sengel C Thony F et al (2008) Place of arterial embolisation in severe blunt hepatic trauma. Cardiovasc Intervent Radiol 31:875-882

Renal Injury

Chow SJD, Thomson KJ, Hartman JF et al (2009) A 10 year review of blunt renal arterial injuries at an urban level 1 trauma centre. Injury 40:844-850

Sofocleous CT, Hinrichs C Hubbi B et al (2005) Angiographic findings and embolotherapy in renal arterial trauma. Cardiovasc Intervent Radiol 28:39-47

Pelvic injury

Agolini SF, Shah K, Jaffe J et al (1997) Arterial embolisation is a rapid and effective technique for controlling pelvic fracture haemorrhage. J Trauma 43:395-397

Tottereman A, Dormagen JB, Madsen JE et al (2006) A protocol for angiographic embolisation in exsanguinating pelvic trauma. Acta Orthop 77:462-468

Travis T, Monsky WL, London J et al (2008) Evaluation of short and long-term complications after emergent internal iliac artery embolisation in patients with pelvic trauma. J Vasc Interv Radiol 9:840-847

Verbeek D, Sugrue M, Balogh Z et al (2008) Acute management of hemodynamically unstable pelvic trauma patients: time for a change? World J Surg 32:1874-1882

Levels of Evidence

Oxford Centre for Evidence-based Medicine (2009) Levels of evidence, 2009. http://www.cebm.net/index.aspx?o= 1025

Oxford Centre for Evidence-based Medicine (2009) Table: Steps in finding evidence ("Levels") for different types of question. http://www.cebm.net/mod_product/ design/files/CEBM-Levels-of-Evidence-2.pdf. 